\title{
Active inclusion of people living with dementia in planning for dementia care and services in Low- and Middle-Income Countries
}

\section{Authors}

1. Erica Breuer, College of Health, Medicine and Wellbeing, University of Newcastle, Australia, ORCID: 0000-0003-0952-6650, erica.breuer@newcastle.edu.au

2. Emily Freeman, Care Policy and Evaluation Centre, London School of Economics and Political Science, UK, ORICD: 0000-0001-9396-1350

3. Suvarna Alladi, National institute of Mental Health and Neurosciences, India, ORCID: 0000-0002-03729572

4. Marinda Breedt, STRiDE South Africa National Advisory Group, South Africa

5. Ishtar Govia, Epidemiology Research Unit, Caribbean Institute for Health Research, The University of the West Indies, Mona Campus, Jamaica, ORCID: 0000-0001-6852-102X

6. Mariana López-Ortega, Research Department, Instituto Nacional de Geriatria, Mexico, ORCID: 00000001-9869-3308

7. Christine Musyimi, Africa Mental Health Research and Training Foundation, Kenya, ORCID: 0000-00028828-2959

8. Déborah Oliveira, Department of Psychiatry, Universidade Federal de São Paulo, Brazil; ORCID: 00000002-6616-533X

9. Meera Pattabiraman, Alzheimer's and Related Disorders Society of India

10. Tara Puspitarini Sani, Alzheimer Indonesia and Atma Jaya Catholic University of Indonesia, Indonesia

11. Marguerite Schneider, Alan J. Flisher Centre for Public Mental Health, University of Cape Town, South Africa, ORCID: 0000-0002-1223-1266

12. Kate Swaffer, Dementia Alliance International \& Faculty of Science, Medicine and Health, University of Wollongong; ORCID: 0000-0001-8963-2431

13. Dubhglas Taylor, Dementia Alliance International and Dementia Awareness Advocacy Team

14. Eileen Taylor, Dementia Alliance International and Dementia Awareness Advocacy Team

15. Adelina Comas-Herrera, Care Policy and Evaluation Centre, London School of Economics and Political Science, UK; ORCID: 0000-0002-9860-9062 


\section{Abstract}

Involving people living with dementia in service design and planning has become more common in high-income countries. It remains rare in low- and middle-income countries where two-thirds of the world's people with dementia live. In this commentary paper, we explore the barriers to inclusion of people living with dementia in planning in low- and middle-income countries and make a case for the inclusion of people living with dementia in care and service planning. We suggest how this can be done at individual, community or national and state level using the following principles: 1) respecting the rights of people living with dementia to self-determination; 2) valuing people living with dementia's unique understanding of dementia; 3) creating a culture of active inclusion which creates a space for people living with dementia to participate; and 4) ensuring appropriate accommodations are in place to maximise participation.

\section{Background}

Ensuring dementia care services are designed by, rather than simply for, people living with dementia is being increasingly supported by both service users and producers. The drive to involve dementia service users in service design and planning has become more common in high-income countries (HICS) (Hanson et al., 2007; Leorin, Stella, Nugent, Cleland, \& Paggetti, 2019; Lorentzon \& Bryan, 2007; Mclntyre, 2003; Penrod et al., 2007; Read, Toye, \& Wynaden, 2020). However, it is rare in lowand middle-income countries (LMICs), where two-thirds of the world's people with dementia live (Prince, 2015). Adequate and appropriate inclusion of people living with dementia in service development respects their right to autonomy and self-determination (making decisions which affect the self) (Kornfeld-Matte, 2015), helps to address unequal power dynamics between service producers (e.g. policy makers, medical practitioners) and users (Lorentzon \& Bryan, 2007), and may ultimately result in more accessible, effective and relevant services (Span, Hettinga, Vernooij-Dassen, Eefsting, \& Smits, 2013). For those directly involved in the planning process, adequate inclusion is expected to help reduce stigmatising behaviours among health and policy practitioners (Abayneh et al., 2017; Gupta \& Roberts, 2014) and encourage those with dementia to feel more empowered (Gupta \& Roberts, 2014; Hagan \& Campbell, 2021) and respected (Whitfield \& Wismer, 2006).

Planning for dementia care and services in many LMICs has been limited by other health priorities and constrained resources (Jamison et al., 2018). Nevertheless, the World Health Organisation's Global Action Plan on the Public Health Response to Dementia 2017-2025, which was adopted at the World Health Assembly in 2017 (World Health Organization, 2017) documents the commitment of LMIC to producing multisectoral National Dementia Plans. Most of these are yet to be developed: by the end of 2020, only six middle-income countries and no low-income countries had published Plans (Alzheimer's Disease International, 2019).

In this commentary paper, we make a case for the inclusion of people living with dementia in developing and operationalising National Dementia Plans in LMICs and provide some specific suggestions for how to achieve this. Although there is little documented on this topic, there have been substantive policy successes in LMICs by people with lived experience of disability. For example, in South Africa, the disability rights movement was instrumental in influencing the South African 
Constitution in 1994 (Howell, Chalklen, \& Alberts, 2006) and people living with HIV were instrumental in advocating for change in access to antiretrovirals for treatment of HIV (Heywood, 2009). The principles which we suggest and operationalise in this paper are based on inclusion research in other areas, such as mental illness, and from lessons learned with regards to dementia in HICs. We also draw on our experiences as people living with dementia (MB, KS, ET), care partners of people living with dementia (IG, MP, DT), members of dementia advocacy organisations $S A, E B, A C H, E F, I G, M L O$, $C M, D O, T P S, K S, M S)$, service providers for people living with dementia $(S A, I G, D O)$, researchers working in the field of dementia, long-term care, and planning for services (SA, EB, ACH, EF, IG, MLO, $C M, D O, T P S, K S, M S$ ), and people with experience of living and working in LMICS (SA, EB, EF, IG, MLO, $D O, M P, T P S, M S)$. Finally, we draw on recent experiences of including people living with dementia in the planning and development of the Strengthening Responses to Dementia in Developing Countries (STRiDE) project (https://stride-dementia.org/) in Brazil, India, Indonesia, Jamaica, Kenya, Mexico and South Africa (E Breuer, Comas-Herrera, Docrat, Freeman, \& Schneider, 2019).

While we recognise the vast heterogeneity of LMICs, we also observe that challenges and solutions associated with including people living with dementia may cut across contexts, including HICs. We also recognise that most of the literature referred to in this commentary paper represents urban rather than rural centres.

\section{Barriers to participation of people living with dementia}

Research on mental health has demonstrated that people with lived experience can contribute to service planning at different levels (Tambuyzer, Pieters, \& Van Audenhove, 2014). For individuals, assuming or contributing to decision-making about one's own care is crucial. At the community level, contributions could include the planning and development of local services, advocacy, guideline development, monitoring and evaluation and research governance. At the state and national levels, participation could range from policy discussions to national level planning of services. However, substantial barriers to inclusion of people living with dementia in LMIC exist at each of these levels.

\subsection{Barriers to individuals' involvement in care decision-making}

Individuals' ability to assume or contribute to decision-making about their care is limited by both late diagnosis and limited opportunity.

Dementia symptoms are often understood to be expected consequences of ageing, (Oliveira et al., 2021) leaving dementia underdiagnosed or diagnosed late. Delayed diagnosis is particularly pronounced in LMICs where families and health and care practitioners are less likely to be aware of dementia or to have received dementia training (Juárez-Cedillo, Jarillo-Soto, \& Rosas-Carrasco, 2014). In Brazil for example, it's estimated that only around $23 \%$ of people living with dementia are diagnosed (Nakamura, Opaleye, Tani, \& Ferri, 2015); in India, that figure is estimated to be less than 10\% (Dias \& Patel, 2009). Individuals experiencing more advanced dementia symptoms are more likely to lack capacity to communicate their needs or make informed choices about their care. In Mexico, evidence suggests that when it happens, dementia diagnosis is frequently late, when cognitive decline and functional impairment is marked (Juárez-Cedillo et al., 2014). 
When dementia is diagnosed, it may not be disclosed (Oliveira et al., 2021). Concerns for people living with dementia may motivate practitioners and families to shield them from difficult information and decisions rather than facilitating their right to autonomy and self-determination (Lepore, Shuman, Wiener, \& Gould, 2017). For example, in Brazil only $44.8 \%$ physicians regularly inform a person living with dementia of their diagnosis (Raicher, Shimizu, Takahashi, Nitrini, \& Caramelli, 2008) and only $58 \%$ of caregivers endorse disclosing the diagnosis (Shimizu, Raicher, Takahashi, Caramelli, \& Nitrini, 2008). In Brazil, as elsewhere (for example, Indonesia, India, Jamaica, Kenya and Mexico), both dominant social norms and laws require adult children to care for vulnerable adults, such as older adults with dementia (Federal Government of Brazil, 2003; Government of India, 2007; Government of Jamaica, 2005; Government of the Republic of Kenya, 2018; Republic of Indonesia, 1971). In doing so, adult children may assume responsibility for medical and financial decisions relating to dementia care (Kristanti et al., 2018).

Structural inequities can also act to limit individuals' involvement in care decisions. For example, literacy, economic and social disadvantage can limit participation (Janic, Kimani, Olembo, \& Dimaras, 2020), as can the design of care systems, including healthcare environments and the adequacy of disability supports (Whitfield \& Wismer, 2006).

\subsection{Barriers to individuals' involvement in community-level care planning}

In many LMICs, dementia is stigmatised (Alzheimer's Disease International, 2019). This stigma is likely to arise within institutionally-based (health service and policy) and community-based social relations, shaping how healthcare practitioners, policy makers and planners respond to and value the voices of people living with dementia (Abayneh et al., 2017) and the willingness of people living with dementia to disclose their diagnosis and risk social exclusion (Alzheimer's Disease International, 2019). Our qualitative research with people living with dementia and their families in Brazil indicates that stigma also manifests in questioning the views and experiences of people living with dementia, leading to people living with dementia internalising this doubt (Oliveira et al., 2021). In the absence of supportive intervention, such people are less likely to volunteer to participate in community-level care planning.

While increasing, organisations which explicitly provide a voice for people living with dementia are not operational in all LMICs. Membership of Dementia Alliance International (DAI), an international organisation of people living with dementia, currently has members in 49 countries, although the majority are HICs (Dementia Alliance International, 2019). Other dementia organisations are often run by caregivers or health practitioner volunteers. Alzheimer's Disease International (ADI) is an international federation of dementia associations with partner organisations in $45 \mathrm{LMICs}$; organisations in $17 \mathrm{LMICs}$ are enrolled in its development programme (L. Dabas, personal communication, $14^{\text {th }}$ August 2020). However, while important in advocating on behalf of persons living with dementia, such organisations do not necessarily include them in developing their advocacy efforts, and service provision is primarily designed to support care givers.

\subsection{Barriers to individuals' involvement in state- or national-level care planning}

As the involvement of people living with dementia in care planning at state/national level is shaped by the barriers to involvement in individual and community level planning, it can be difficult to identify 
people who have been diagnosed and still have the capacity to communicate their needs to participate or whose families support them to participate. In addition, stigmatisation of dementia may also arise among people working at these higher planning levels. This, along with limited support for people living with cognitive disabilities in LMICs (World Health Organisation, 2019), may lead to the underestimation of the capacity of persons with dementia to contribute meaningfully (Swaffer, 2014).

Participation of people living with dementia in state/national level care planning is made more difficult because in most LMICs dementia care is fragmented. In addition, comprehensive multidisciplinary and multisectoral dementia care policies or programs that include diagnosis and support/treatment are rare (Alzheimer's Disease International, 2019). In most settings, the majority of dementia care is provided by families, other informal paid or unpaid providers, and the private sector (Prince, 2004). Until more recently, dementia care planning has not been a priority in the face of other significant and urgent needs for health and social services at the population level (e.g. high levels of infectious disease, maternal and infant mortality, non-communicable diseases, and violence) (Jamison et al., 2018).

When dementia care and services are being planned, dementia-specific structures or processes to encourage participation may not exist. For example, in Brazil 'social control' systems for eliciting public contributions to health policy and planning are well-established (Noronha \& Castro, 2019); municipal, state and national Councils of Older Persons facilitate older adults' contributions (Veras \& Oliveira, 2018). However, none of these are dementia-specific or routinely include mechanisms to support participation of people living with dementia or other people with mental disabilities.

\section{Pathways to the inclusion of people living with dementia}

We recommend that the inclusion of people living with dementia in care planning should be guided by the following key principles, at individual, community, or national and state level:

1. Respecting the rights of people living with dementia to self-determination

2. Valuing people living with dementia's unique understanding of dementia

3. Creating a culture of active inclusion which creates a space for people living with dementia to participate

4. Ensuring appropriate accommodations are in place to maximise participation

These principles are not unique to people living with dementia and can be relevant to other disabilities. However, we show how they can be applied specifically to people living with dementia. At the individual level, we anticipate that they will primarily be used by care providers. These include social and healthcare providers of both formal and unpaid care. At the community level, the principles can be applied dementia organisations, people planning local health and social care services, and those conducting research with people living withresearching dementia. At the state/national level, the principles are likely to be applied by policy makers and planners.

More detail of practical ways to implement these principles are provided as follows below and in Table 1. 
Table 1 Strategies to ensure active inclusion

\begin{tabular}{|c|c|c|c|}
\hline Principles & Individual & Community & State/national \\
\hline $\begin{array}{l}\text { Right to self } \\
\text { determination }\end{array}$ & $\begin{array}{l}\text { - Provide person centred care which } \\
\text { involves people with dementia in } \\
\text { decision making about their care } \\
\text { (Lorentzon \& Bryan, 2007) } \\
\text { - } \quad \text { Promote human rights protection } \\
\text { (Abayneh et al., 2020) } \\
\text { - Disclose dementia diagnosis to the } \\
\text { person living with dementia }\end{array}$ & 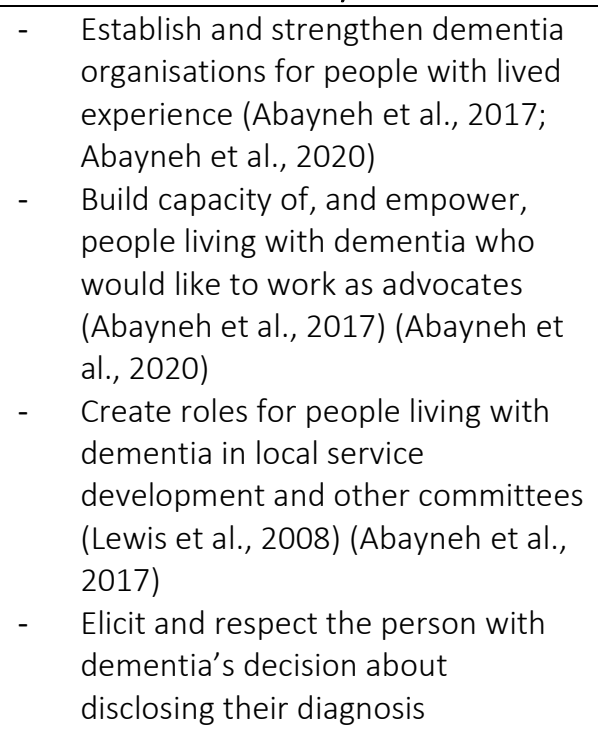 & 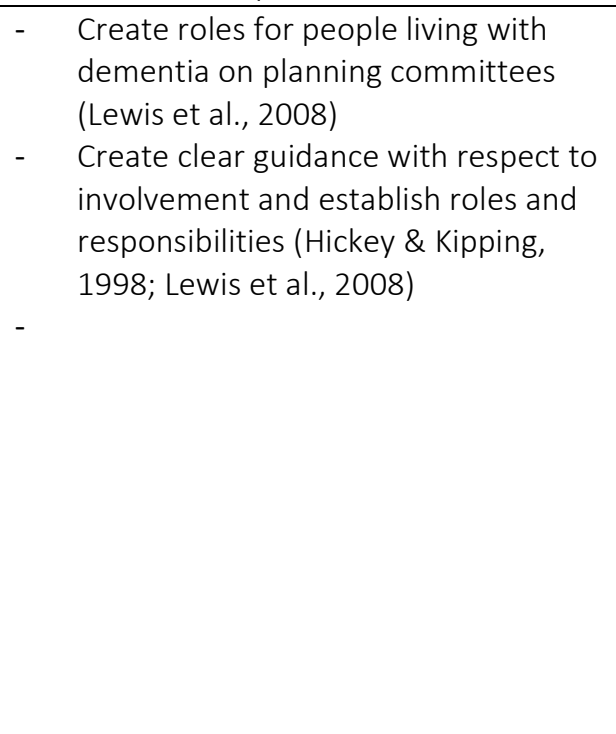 \\
\hline $\begin{array}{l}\text { Valuing people living } \\
\text { with dementia as } \\
\text { experts }\end{array}$ & $\begin{array}{l}\text { Develop shared care plans and allow } \\
\text { the person with dementia to influence } \\
\text { decisions (Daly, Bunn, \& Goodman, } \\
\text { 2018), within both formal and } \\
\text { informal contexts of care. }\end{array}$ & 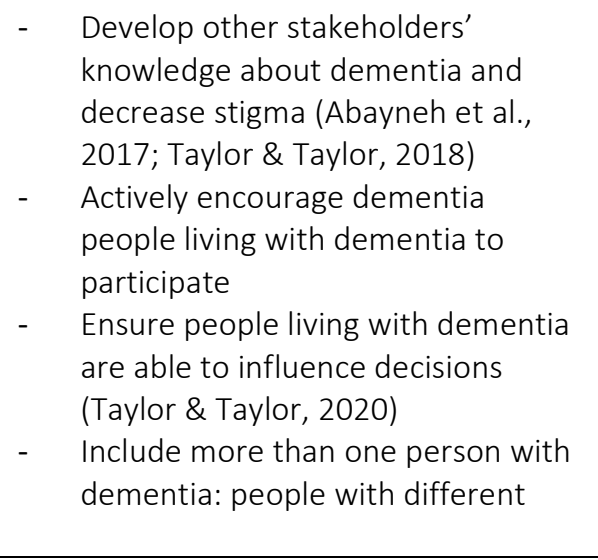 & 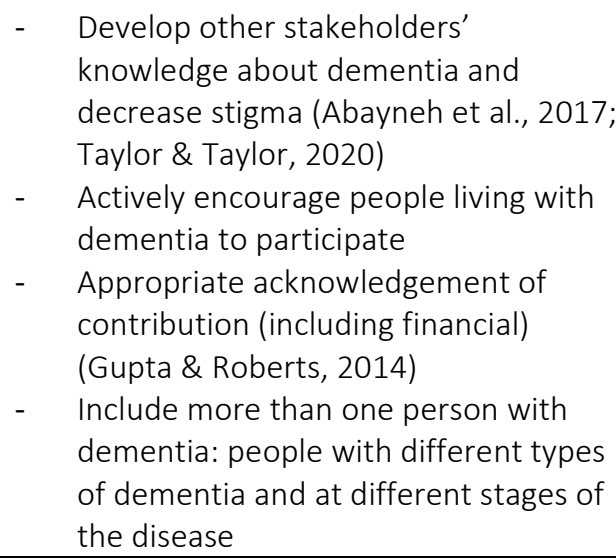 \\
\hline
\end{tabular}


types of dementia and at different

stages of the disease

\section{Culture of active}

inclusion

Establish groups for people living with

dementia (Abayneh et al., 2020)

Ensuring appropriate accommodations
- $\quad$ Encourage person with dementia to speak for themselves (Goeman et al., 2019; Taylor \& Taylor, 2020)

- $\quad$ Active and respectful listening

(Taylor \& Taylor, 2020)

- $\quad$ Use dementia inclusive language (Swaffer, 2014)

Use practical strategies outlined in Table 2 depending on the needs of the person(s) living with dementia
Use practical strategies outlined in Table 2 depending on the needs or preferences of the person living with dementia

- $\quad$ Include funding in budget (for time and disability support as well as travel and accommodation)

- Ensure people living with dementia able to influence decisions (Taylor \& Taylor, 2020)

- $\quad$ Respect the person with dementia's choice about whether they wish to disclose diagnosis

Use dementia inclusive language (Swaffer, 2014)

se practical strategies outlined in Table 2 depending on the needs of the person(s) living with dementia 


\subsection{Facilitating individuals' involvement in care decision-making}

Both structural (e.g. extent, nature and functioning of health systems; stigmatisation of dementia) and local (e.g. family level) changes are necessary to facilitate the rights of people living with dementia to decide on their own care. Some changes are significant and will require a wide-ranging redesign; others could be achieved with more bounded interventions.

For example, introducing shared care plans at a service level may first require steps to both, decrease stigmatisation of dementia among family members and care practitioners, and shift understandings of the often hierarchical 'patient' and care practitioner roles more broadly, to ensure that these gatekeepers value the voice of the person living with dementia in determining their own care pathway. However, it some settings, it would also require the implementation of a clearer care pathway to dementia care (e.g. from primary care to specialist dementia care) in which to introduce shared care plans. In Mexico for instance, there is no unique first point of contact in the healthcare system; no specific sector or 'level' that provides dementia care. Here, the implementation of shared care plans would first require identification (training and awareness-raising) of the appropriate 'service level' such as general practitioners, social workers, and gerontologists.

On the other hand, introducing shared care plans at a family level - where the vast majority of care is provided - may be more straightforward to operationalise. While formal publicly funded strategies are designed and implemented, accessible information and training about dementia, its possible progression and options for non-pharmacological management could help unpaid, frequently family, caregivers to become care partners. This, alongside wider anti-stigma interventions, would help families and others to be as prepared as possible to care for the person living with dementia, and plan care pathways with them in a way that takes their needs and preferences into account.

Timely diagnosis of dementia, and disclosure of the diagnosis to the person living with dementia, is a key precursor to facilitating shared care planning at both levels. Increasing public awareness of dementia symptoms and their distinction from 'normal ageing', as well as steps to reduce the stigmatisation of dementia through community based initiatives (Phillipson et al., 2019), could facilitate timely diagnosis by encouraging people with early signs of cognitive impairment and their family members to recognise these signs and seek professional help.

Once a dementia diagnosis has been received and accepted by the person living with dementia, it is possible to contribute developing shared care plans (Daly, Bunn et al. 2018). Our ongoing research and care consultations in Jamaica, indicates the need for gentle and gradual building of a partnership care models over time, in order to counter resistance of those believed to have dementia to seek healthcare or accept the possibility of a dementia diagnosis. Establishing peer support groups for people living with dementia may additionally support this by increasing social inclusion and reducing isolation (Dam, de Vugt, Klinkenberg, Verhey, \& van Boxtel, 2016). 


\subsection{Facilitating individuals' involvement in community level care planning}

Establishing or strengthening advocacy organisations which are led by and for people with dementia could be key to facilitating involvement in community-level care planning (Lempp et al., 2018). These organisations help build capacity and support people living with dementia who are willing to work as advocates, and can help service planners and researchers to identify people living with dementia who are willing and able to contribute to service planning (McConnell et al., 2018). This is important because only a subset of people living with dementia may want to contribute to dementia services and planning, particularly in public fora and in the contexts where dementia is highly stigmatised. For example, despite our best efforts, we were only able to involve people living with dementia in five of seven of our national level STRiDE research planning workshops(Erica Breuer et al., 2021). These consultative workshops included various stakeholders and asked them to map out what needed to be in place to improve dementia care and services in each of the STRiDE countries(Erica Breuer et al., 2021). In Mexico and Indonesia, we invited participation of people living with dementia identified through Alzheimer's associations and physicians providing specialised dementia care but could not find anyone willing to participate. Reasons for not participating included feeling shame about dementia or fear of disclosure, not feeling able to contribute or family members not wanting to put the person living with dementia under excessive stress.

In planning processes, a role needs to be created for people living with dementia to participate in local service development and other committees (Lewis et al., 2008). This includes ensuring people living with dementia are aware of the planning processes and ways in which they can participate. In the service development process, there should be a culture of inclusion so that people living with dementia are encouraged to speak for themselves, are actively and respectfully listened to and are able to influence decisions (Whitfield \& Wismer, 2006). To ensure a culture of inclusion, explicit strategies might be required such as increasing stakeholders' knowledge of dementia, decreasing stigma, and changing the ways of working such as using disability supports (Table 2).

While facilitating the inclusion of multiple people living with dementia in any given planning process will obviously lead to better and more informed outputs, having more than one person living with dementia involved may additionally provide peer support and encouragement that would increase the ability of each person to participate and thus strengthen the quality of individual contributions. People living with dementia should be able to participate in community-level planning processes anonymously or without disclosing their diagnosis. This worked well in our research planning workshop in Jamaica where participants were introduced by name but not role, leaving those with dementia to disclose their diagnosis if and when they wanted to. As the workshop progressed, one participant who was initially reluctant to disclose felt empowered to share her own experience and has since shared her experience in another community-level dementia-related forum. 


\subsection{Facilitating individuals' involvement in state- or national-level care planning}

Planning processes, particularly at state/national levels, should have clear strategic pathways for including people living with dementia (Lewis et al., 2008). This should include guidance for establishing their roles and responsibilities (Hickey \& Kipping, 1998; Lewis et al., 2008) and doing so in a manner that facilitates their involvement as the disease progresses, including providing disability support (Swaffer, 2014). People living with dementia should be appropriately acknowledged for their contribution including through payment - especially if other experts are paid (Gupta \& Roberts, 2014).

Given the shared experience of some elements of living with dementia, where it is not possible to find someone local to include in state/national level planning processes, for example because of fear or stigma, experience from DAl has shown that it may be helpful to initially invite a person living with dementia from another setting to participate. DAl found that the inclusion of people living with dementia as invited keynote speakers or delegates at conferences or participants in focus groups for research, gives courage to those with dementia who have not had a voice to come forward to advocate for themselves.

\subsection{Access to disability supports}

At all three levels of care planning, people living with dementia should have access to disability supports related to their individual needs. These supports, highlighted in Table 2, include assistance with communication, rehabilitation, physical environments, mental well-being, carers and continued engagement. These are based on literature, our experience with STRiDE and working with people living with dementia. Budgets for planning processes should include these disability supports.

Table 2 Disability supports which can be adapted for individual needs

\begin{tabular}{lll}
\hline Needs & & \multicolumn{1}{c}{ Disability supports } \\
\hline Communication & - & Prepare and share materials prior to the meeting \\
- & Materials should be in plain language, simple to understand and visually \\
& appealing with large font sizes (Northway, Howarth, \& Evans, 2015) (Goeman et \\
& al., 2019) \\
- & Pay attention and adapt materials to literacy and health literacy \\
- & Arrange a practice/orientation session and follow up session for person living \\
& with dementia for important meetings \\
- & Have clear and simply communicated tasks and items on which decisions need to \\
& be made \\
- & Use clear language and avoid jargon (Abayneh et al., 2017; Lorentzon \& Bryan, \\
& 2007) \\
- & Consider shorter but more frequent meetings \\
- & Ensure questions can be asked throughout \\
- & Check for understanding \\
- & Make allowance for 'wrong' ideas and words (Lorentzon \& Bryan, 2007) \\
- & Use specific communication tools designed for people living with dementia \\
& (Wang, Marradi, Albayrak, \& Van Der Cammen, 2019)
\end{tabular}




\begin{tabular}{|c|c|}
\hline $\begin{array}{l}\text { Physical } \\
\text { environment }\end{array}$ & $\begin{array}{ll}\text { - } & \text { Ensure physically accessible location for persons with mobility restrictions } \\
\text { - } & \text { Ensure safe travel to venue available (and reimburse for travel) (Goeman et al., } \\
\text { 2019) } \\
\text { - } \quad \text { Consider number of people in the room(Wang et al., 2019) } \\
\text { - } \quad \text { Keep noise levels down (Wang et al., 2019) } \\
\text { - } \quad \text { Ensure clear signage } \\
\text { - } & \text { Have a practice session for virtual meeting platforms with person with dementia }\end{array}$ \\
\hline $\begin{array}{l}\text { Mental well- } \\
\text { being }\end{array}$ & $\begin{array}{l}\text { - } \quad \text { Ensure the person with dementia is comfortable (Lorentzon \& Bryan, 2007) } \\
\text { - Assign a psychologist or support person to the person living with dementia to } \\
\text { monitor the needs, provide one on one support and answer questions for } \\
\text { clarification }\end{array}$ \\
\hline $\begin{array}{l}\text { Caregiver or } \\
\text { care partner }\end{array}$ & $\begin{array}{l}\text { - } \quad \text { Provide funding for a caregiver or care partner to travel with the person living } \\
\text { with dementia } \\
\text { - } \quad \text { Allow opportunities to contribute without caregiver or care partner }\end{array}$ \\
\hline $\begin{array}{l}\text { Continued } \\
\text { engagement }\end{array}$ & $\begin{array}{l}\text { - } \quad \text { Create a plan for continued engagement which takes into account disease } \\
\text { progression (Whitfield \& Wismer, 2006) } \\
\text { - Be flexible with alternative options for engagement (e.g. via phone call, individual } \\
\text { meeting) and reschedule if necessary }\end{array}$ \\
\hline
\end{tabular}

\section{Conclusion}

In this commentary, we have presented some challenges related to the active inclusion of people living with dementia in planning for services in LMIC and provided a set of principles and concrete strategies to guide inclusion. We recognise that not all strategies may be feasible to implement or necessary in every LMIC. Structural barriers to inclusion such as the political context, stigma and health systems need to be addressed. Meaningful inclusion is a long-term investment and requires sincere engagement from all stakeholders involved.

\section{Keywords}

Dementia, planning, low- and middle-income countries, inclusion, patient involvement

\section{Declaration of Interest}

The authors declare no competing interests.

\section{Funding}

This work was supported by the UK Research and Innovation's Global Challenges Research Fund (ES/P010938/1) 


\section{References}

Abayneh, S., Lempp, H., Alem, A., Alemayehu, D., Eshetu, T., Lund, C., .. Hanlon, C. (2017). Service user involvement in mental health system strengthening in a rural African setting: qualitative study. $B M C$ Psychiatry, 17(1), 187. doi:10.1186/s12888-017-1352-9

Abayneh, S., Lempp, H., Alem, A., Kohrt, B. A., Fekadu, A., \& Hanlon, C. (2020). Developing a Theory of Change model of service user and caregiver involvement in mental health system strengthening in primary health care in rural Ethiopia. Int J Ment Health Syst, 14, 51. doi:10.1186/s13033-020-00383-6

Alzheimer's Disease International. (2019). Dementia plans. Retrieved from https://www.alz.co.uk/dementiaplans

Breuer, E., Comas-Herrera, A., Docrat, S., Freeman, E., \& Schneider, M. (2019). STRiDE Theory of Change Workshops: Guidance and Resources. STRIDE Research Tool No.1 (version 2). Retrieved from https://www.stride-dementia.org/publications

Breuer, E., Comas-Herrera, A., Freeman, E., Albanese, E., Alladi, S., Amour, R., . . I Iveth Astudillo García, C. (2021). Beyond the project: Building a strategic theory of change to address dementia care, treatment and support gaps across seven middle-income countries. Dementia, 14713012211029105.

Daly, R. L., Bunn, F., \& Goodman, C. (2018). Shared decision-making for people living with dementia in extended care settings: a systematic review. BMJ open, 8(6), e018977. doi:10.1136/bmjopen-2017-018977

Dam, A. E. H., de Vugt, M. E., Klinkenberg, I. P. M., Verhey, F. R. J., \& van Boxtel, M. P. J. (2016). A systematic review of social support interventions for caregivers of people with dementia: Are they doing what they promise? Maturitas, 85, 117-130. doi:https://doi.org/10.1016/j.maturitas.2015.12.008

Dementia Alliance International. (2019). https://www.dementiaallianceinternational.org/where-in-the-world-isdai/.

Dias, A., \& Patel, V. (2009). Closing the treatment gap for dementia in India. Indian J Psychiatry, 51(Suppl1), S93.

Elderly Statute 2003 (Brazil),, Law 10,741/2003 C.F.R. (2003).

Goeman, D. P., Corlis, M., Swaffer, K., Jenner, V., Thompson, J. F., Renehan, E., \& Koch, S. (2019). Partnering with people with dementia and their care partners, aged care service experts, policymakers and academics: A co-design process. Australasian Journal on Ageing, 38, 53-58. Retrieved from https://onlinelibrary.wiley.com/doi/pdfdirect/10.1111/ajag.12635?download=true

Government of India. (2007). Maintenance and Welfare of Parents and Senior Citizens Act, 2007. Gazette of India, 31.

Maintenance Act (Jamaica), (2005) The care and protection of older members of society bill, PART III 59(f) C.F.R. (2018).

Gupta, E., \& Roberts, B. (2014). User and researcher collaborations in mental health in low and middle income countries: a case study of the EMPOWER project. BMC Res Notes, 7, 37. doi:10.1186/1756-0500-7-37

Hagan, R. J., \& Campbell, S. (2021). Doing their damnedest to seek change: How group identity helps people with dementia confront public stigma and maintain purpose. Dementia, 1471301221997307.

Hanson, E., Magnusson, L., Arvidsson, H., Claesson, A., Keady, J., \& Nolan, M. (2007). Working together with persons with early stage dementia and their family members to design a user-friendly technologybased support service. Dementia, 6(3), 411-434.

Heywood, M. (2009). South Africa's Treatment Action Campaign: Combining Law and Social Mobilization to Realize the Right to Health. Journal of Human Rights Practice, 1(1), 14-36. doi:10.1093/jhuman/hun006

Hickey, G., \& Kipping, C. (1998). Exploring the concept of user involvement in mental health through a participation continuum. J Clin Nurs, 7(1), 83-88.

Howell, C., Chalklen, S., \& Alberts, T. (2006). A history of the disability rights movement in South Africa. Disability and social change: A South African agenda, 46-84.

Jamison, D. T., Alwan, A., Mock, C. N., Nugent, R., Watkins, D., Adeyi, O., .. Z Zhao, K. (2018). Universal health coverage and intersectoral action for health: key messages from Disease Control Priorities, 3rd edition. The Lancet, 391(10125), 1108-1120. doi:https://doi.org/10.1016/S0140-6736(17)32906-9

Janic, A., Kimani, K., Olembo, I., \& Dimaras, H. (2020). Lessons for Patient Engagement in Research in Low- and Middle-Income Countries. Ophthalmol Ther, 9(2), 221-229. doi:10.1007/s40123-020-00246-w 
Juárez-Cedillo, T., Jarillo-Soto, E. C., \& Rosas-Carrasco, O. (2014). Social representation of dementia and its influence on the search for early care by family member caregivers. American Journal of Alzheimer's Disease \& Other Dementias ${ }^{\circledR}, 29(4), 344-353$.

Kornfeld-Matte, R. (2015). Report on autonomy and care (A/HRC/30/43), submitted by the Independent Expert on on the enjoyment of all human rights by older persons. Retrieved from

https://www.un.org/development/desa/ageing/news/2016/09/report-of-the-independent-expert-onthe-enjoyment-of-all-human-rights-by-older-persons/

Kristanti, M. S., Engels, Y., Effendy, C., Astuti, Utarini, A., \& Vernooij-Dassen, M. (2018). Comparison of the lived experiences of family caregivers of patients with dementia and of patients with cancer in Indonesia. Int Psychogeriatr, 30(6), 903-914. doi:10.1017/S1041610217001508

Lempp, H., Abayneh, S., Gurung, D., Kola, L., Abdulmalik, J., Evans-Lacko, S., . . Hanlon, C. (2018). Service user and caregiver involvement in mental health system strengthening in low- and middle-income countries: a cross-country qualitative study. Epidemiol Psychiatr Sci, 27(1), 29-39. doi:10.1017/S2045796017000634

Leorin, C., Stella, E., Nugent, C., Cleland, I., \& Paggetti, C. (2019). The value of including people with dementia in the co-design of personalized eHealth technologies. Dement Geriatr Cogn Disord, 47(3), 164-175.

Lepore, M., Shuman, S. B., Wiener, J. M., \& Gould, E. (2017). Challenges in involving people with dementia as study participants in research on care and services. Research summit om dementia care. Building evidence for services and supports.

Lewis, A., Parsons, S., Robertson, C., Feiler, A., Tarleton, B., Watson, D., . . Marvin, C. (2008). Participation in Research: Reference, or advisory, groups involving disabled people: reflections from three contrasting research projects. British Journal of Special Education, 35(2), 78-84.

Lorentzon, M., \& Bryan, K. (2007). Respect for the person with dementia: fostering greater user involvement in service planning. Quality in Ageing and Older Adults.

McConnell, T., Best, P., Sturm, T., Stevenson, M., Donnelly, M., Taylor, B. J., \& McCorry, N. (2018). A translational case study of empowerment into practice: A realist evaluation of a member-led dementia empowerment service. Dementia, 19(6), 1974-1996. doi:10.1177/1471301218814393

Mclntyre, M. (2003). Dignity in dementia: Person-centered care in community. Journal of Aging Studies, 17(4), 473-484.

Nakamura, A. E., Opaleye, D., Tani, G., \& Ferri, C. P. (2015). Dementia underdiagnosis in Brazil. The Lancet, 385(9966), 418-419.

Noronha, J. C. d., \& Castro, L. (2019). Democracy, Health, and the 16th National Health Conference in Brazil: what future? Cadernos de saude publica, 35.

Northway, R., Howarth, J., \& Evans, L. (2015). Participatory research, people with intellectual disabilities and ethical approval: making reasonable adjustments to enable participation. J Clin Nurs, 24(3-4), 573-581. doi:https://doi.org/10.1111/jocn.12702

Oliveira, D., Da Mata, F. A. F., Mateus, E., Musyimi, C. W., Farina, N., Ferri, C. P., \& Evans-Lacko, S. (2021). Experiences of stigma and discrimination among people living with dementia and family carers in Brazil: qualitative study. Ageing and Society, 1-22. doi:10.1017/s0144686x21000660

Penrod, J., Yu, F., Kolanowski, A., Fick, D. M., Loeb, S. J., \& Hupcey, J. E. (2007). Reframing person-centered nursing care for persons with dementia. Research and theory for nursing practice, 21(1), 57-72.

Phillipson, L., Hall, D., Cridland, E., Fleming, R., Brennan-Horley, C., Guggisberg, N., . . Hasan, H. (2019). Involvement of people with dementia in raising awareness and changing attitudes in a dementia friendly community pilot project. Dementia, 18(7-8), 2679-2694. doi:10.1177/1471301218754455

Prince, M. (2004). Care arrangements for people with dementia in developing countries. International Journal of Geriatric Psychiatry.

Prince, M. (2015). World Alzheimer Report 2015: the global impact of dementia: an analysis of prevalence, incidence, cost and trends. Retrieved from

Raicher, I., Shimizu, M. M., Takahashi, D. Y., Nitrini, R., \& Caramelli, P. (2008). Alzheimer's disease diagnosis disclosure in Brazil: a survey of specialized physicians' current practice and attitudes. International Psychogeriatrics, 20(3), 471-481. doi:10.1017/S1041610207005819

Read, S. T., Toye, C., \& Wynaden, D. (2020). The participation of people with dementia in the planning of their care and support: An integrative literature review. Dementia, 19(3), 691-707.

Indonesian Marriage Law no. 1/1974 (article 46), (1971).

Shimizu, M. M., Raicher, I., Takahashi, D. Y., Caramelli, P., \& Nitrini, R. (2008). Disclosure of the diagnosis of Alzheimer's disease: caregivers' opinions in a Brazilian sample. Arq Neuropsiquiatr, 66, 625-630. 
Retrieved from http://www.scielo.br/scielo.php?script=sci arttext\&pid=S0004-

$\underline{282 \times 2008000500004 \& n r m=i s 0}$

Span, M., Hettinga, M., Vernooij-Dassen, M., Eefsting, J., \& Smits, C. (2013). Involving people with dementia in the development of supportive IT applications: A systematic review. Ageing Research Reviews, 12(2), 535-551. doi:10.1016/j.arr.2013.01.002

Swaffer, K. (2014). Dementia: Stigma, Language, and Dementia-friendly. Dementia, 13(6), 709-716. doi:10.1177/1471301214548143

Tambuyzer, E., Pieters, G., \& Van Audenhove, C. (2014). Patient involvement in mental health care: one size does not fit all. Health Expectations, 17(1), 138-150.

Taylor, E., \& Taylor, D. A. (2018). Inclusion is a Right: Together we achiev more. Paper presented at the Alzehimer's New Zealand Conference, Auckland.

Taylor, E., \& Taylor, D. A. (2020). Even so, rights, if it has no action, is dead: Don't talk - ACT, don't say - DO, don't promise - PROVE! . In L. Steele, K. Swaffer, L. Phillipson, \& R. Fleming (Eds.). Sydney: University of Technology Sydney.

Veras, R. P., \& Oliveira, M. (2018). Envelhecer no Brasil: a construção de um modelo de cuidado. Ciência \& Saúde Coletiva, 23, 1929-1936.

Wang, G., Marradi, C., Albayrak, A., \& Van Der Cammen, T. J. M. (2019). Co-designing with people with dementia: A scoping review of involving people with dementia in design research. Maturitas, 127, 5563. doi:10.1016/j.maturitas.2019.06.003

Whitfield, K., \& Wismer, S. (2006). Inclusivity and dementia: health services planning with individuals with dementia: effective inclusion requires action at multiple levels by individuals with dementia, care partners, service providers and funding organizations. Healthcare Policy, 1(2), 120.

World Health Organisation. (2019). Dementia Fact Sheets. Retrieved from https://www.who.int/newsroom/fact-sheets/detail/dementia

World Health Organization. (2017). Global action plan on the public health response to dementia 2017-2025. 